\title{
QUADRUPOLAR MAGNETIC FIELD OUTSIDE ANTIFERROMAGNETIC $\mathrm{Cr}_{2} \mathrm{O}_{3}$
}

\author{
A.S. Borovik-Romanov and V.I. NIZHankovskiI \\ International Laboratory of High Magnetic Fields and Low Temperatures \\ Gajowicka 95, 53-529 Wrocław, Poland \\ and
}

P.L. Kapitza Institute for Physical Problems, Kosygin str. 2, 117334 Moscow, Russia

Predicted by Dzyaloshinskii quadrupolar magnetic field outside antiferromagnet was experimentally observed for $\mathrm{Cr}_{2} \mathrm{O}_{3}$ single crystal. The magnetic quadrupole sharply disappeared with heating the sample above $T_{\mathrm{N}}=$ $317 \mathrm{~K}$ and appeared again on cooling below $T_{\mathrm{N}}$. The obtained value of the magnetic quadrupole is in reasonable agreement with theoretical estimation.

PACS numbers: 75.10.-b, 75.25.+z, 75.50.Ee

Recently Dzyaloshinskii [1] has pointed out that antiferromagnets which display the magnetoelectric effect (this magnetic structure does not possess the centre of symmetry) should produce quadrupolar magnetic field. Astrov and Ermakov [2] have confirmed this prediction experimentally for $\mathrm{Cr}_{2} \mathrm{O}_{3}$ single crystal. In the present paper wc report the results obtained on the same sample in wide temperature range from 4.2 to $324 \mathrm{~K}$ using different from [2] method of measurements.

First of all we present formulas for the magnetic field and flux produced by quadrupole (coefficients given by Dzyaloshinskii in [1] are not correct). Generally, a quadrupole consists of two opposite dipoles $m,-m$ displaced by vector $d$. In the dipole approximation

$$
H=\frac{3(m r) r}{r^{5}}-\frac{m}{r^{3}}-\frac{3[m(r+d)](r+d)}{|r+d|^{5}}+\frac{m}{|r+d|^{3}},
$$

where $r$ is the vector from an observer to the dipole $m$. We find radial component of $\boldsymbol{H}$ by multiplying Eq. (1) by $r / r$ and neglecting terms with $d^{2}$ :

$$
H_{r}=\frac{3}{r^{6}}\left[3(m r)(d r)-(m d) r^{2}\right] .
$$

For the case $m \| d$

$$
H_{r}=\frac{3(m d)}{r^{4}}\left(3 \cos ^{2} v-1\right),
$$

where $v$ is the angle between $\boldsymbol{r}$ and $\boldsymbol{d}$ (the quadrupole axis). 
Now we may calculate flux through a coil. If the quadrupole is placed at coil's axis at a distance $z$ from its centre and $\varphi$ is the angle between quadrupole's and coil's axes then the answer is

$$
\Phi(z, \varphi)=3 \pi a^{2}(m d) \frac{z}{\left(z^{2}+a^{2}\right)^{5 / 2}}(3 \cos \varphi-1),
$$

where $a$ is the radius of a coil. From this formula it can be seen that at given $z$ the flux for $\varphi=90^{\circ}$ has an opposite sign and is exactly twice smaller than for $\varphi=0^{\circ}$.

Let us estimate the quadrupole ( $m d)$ for $\mathrm{Cr}_{2} \mathrm{O}_{3}$ from structural data. $\mathrm{Cr}_{2} \mathrm{O}_{3}$ has rhombohedral unit cell (Fig. 1a). Magnetic $\mathrm{Cr}^{3+}$ ions have positions at $u h$, $(1 / 2-u) h,(1 / 2+u) h,(1-u) h$ along the trigonal axis $C_{3}$ with a length

$$
h=3 a \sqrt{1-\frac{4}{3} \sin ^{2} \frac{\alpha}{2}},
$$

where $u=0.145, a=5.38 \AA, \alpha=54^{\circ} 50^{\prime}$. Quadrupole of the cell is

$$
\begin{aligned}
& (m d)_{\text {cell }}=g \mu_{\mathrm{B}} S[-u+(1 / 2-u)-(1 / 2+u)+(1-u)] h \\
& \quad=g \mu_{\mathrm{B}} S(1-4 u) h,
\end{aligned}
$$

where $g$ is the Landé factor, $\mu_{\mathrm{B}}$ is the Bolrr magneton. Quadrupole of the sample is

$$
(m d)=N_{\mathrm{A}} \frac{m_{\text {sample }}}{2 M}(m d)_{\text {cell }} \text {, }
$$

where $N_{\mathrm{A}}$ is the Avogadro number, $m_{\text {sample }}$ is the mass of the sample, $M$ is $\mathrm{Cr}_{2} \mathrm{O}_{3}$ molecular mass (unit cell contains two formula units). Taking $g=2, S=3 / 2$ and $m_{\text {sample }}=0.7187 \mathrm{~g}$ we get $(m d)_{\text {cell }}=1.0645 \times 10^{-27} \mathrm{G} \mathrm{cm}^{4}$ and $(m d)=$ $1.51 \times 10^{-6} \mathrm{G} \mathrm{cm}$.

Our device (Fig. 1b) consisted of pick-up coil (two turns from Nb-Ti wire joined in opposite) connected to DC SQUID sensor. Distance between the turns was $4 \mathrm{~cm}$ and their radius $a=1.2 \mathrm{~cm}$. The coil was placed outside a dewar-insert made from glass. Inside it a tube glued from aluminium foil and paper with bifilar heater and Si-diode thermometer was mounted. Temperature was stabilised manually with an accuracy about $1 \mathrm{~K}$. The main cryostat was made from fiberglass and shielded with three thin-wall permalloy tubes. The middle tube has longitudinal demagnetising coil, the inner one - longitudinal and toroidal demagnetising coils. No superconducting shield was used, even for SQUID sensor. Residual magnetic
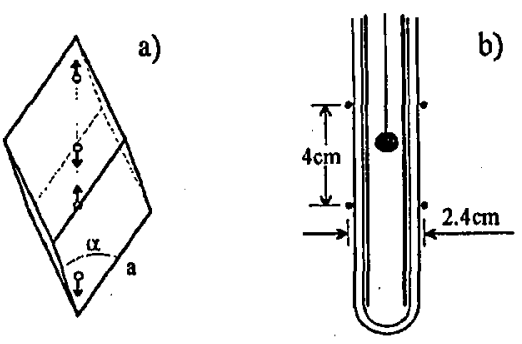

Fig. 1. (a) Crystallographic structure of $\mathrm{Cr}_{2} \mathrm{O}_{3}$. (b) Schematic of the experimental setup. 


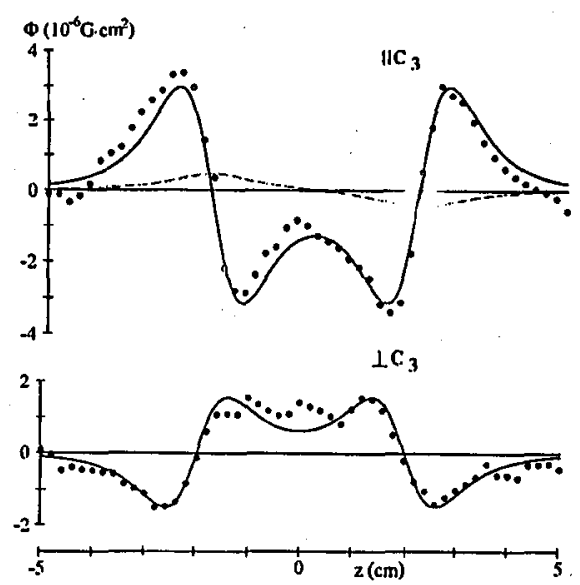

Fig. 2. Magnetic flux registered from $\mathrm{Cr}_{2} \mathrm{O}_{3}$ sample at $T=4.2 \mathrm{~K}$ when the sample is moved along the pickup coil's axis. Solid lines are the least squares fits to Eq. (7), dashed line at upper panel shows a dipole contribution.

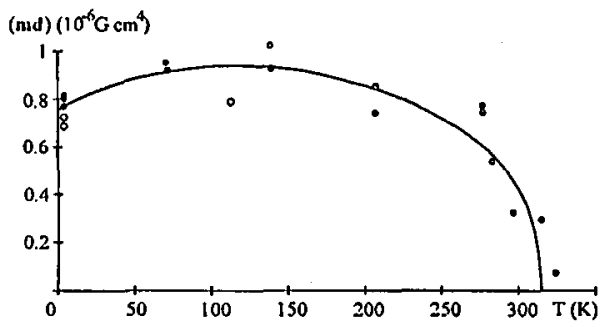

Fig. 3. Temperature dependence of the quadrupole of $\mathrm{Cr}_{2} \mathrm{O}_{3}$ sample. Experimental points were obtained for parallel $(0)$ and perpendicular $(\bullet)$ orientations of the trigonal axis of the sample respectively to the coil's axis. Solid line is a guide for eyes.

field at the sample position did not exceed $3 \times 10^{-4} \mathrm{Oe}$. The sample was ball-shaped single crystal $\mathrm{Cr}_{2} \mathrm{O}_{3} 0.63 \pm 0.01 \mathrm{~cm}$ in diameter. The sample was placed in a basket made from thin $0.1 \mathrm{~cm}$ wide paper tape and suspencled with a cotton thread $65 \mathrm{~cm}$ long. No glue was used in preparation of this sample's holder. All these precautions permitted us to avoid parasitic magnetic signals. We moved the sample along the coil's axis and registered the change in fux as a function of distance. Calibration of the device was performed with known magnetic flux produced by dipole and quadrupole coils with dimensions similar to those of the sample.

In Fig. 2 the results obtained at $T=4.2 \mathrm{~K}$ for parallel $\left(\varphi=0^{\circ}\right)$ and perpendicular $\left(\varphi=90^{\circ}\right)$ orientations of the trigonal axis of the sample respectively to the coil's axis are shown. Solid lines are the least squares fit of the experimental points to the function

$$
\Phi_{\mathrm{s}}(z, \varphi)=\Phi(z+2 \mathrm{~cm}, \varphi)-\Phi(z-2 \mathrm{~cm}, \varphi)
$$


where $\Phi(z, \varphi)$ is given by Eq. (4). As it is seen, the results nicely correspond to the expected quadrupole behaviour except that small dipole contribution is visible for parallel orientation. The same measurements were performed at higher temperatures. It was seen that quadrupole keeps up to the Neel point $\left(T_{\mathrm{N}}=\right.$ $317 \mathrm{~K})$, disappears above $T_{\mathrm{N}}$ and arises again with cooling below $T_{\mathrm{N}}$. All measured curves were fitted to $\Phi_{\mathrm{s}}$ (Eq. (7)) and the dependence of $(\boldsymbol{m d})$ on temperature was obtained, see Fig. 3. The observed maximal value of quadrupole was only 1.5 times smaller than estimated with Eq. (6).

In conclusion we must emphasise that such measurements of magnetic quadrupole are an unique way for determining the configuration of spins in the antiferromagnetic state.

\section{References}

[1] I. Dzyaloshinskii, Solid State Commun. 82, 579 (1992).

[2] D.N. Astrov, N.B. Ermakov, JETP Lett. 59, 274 (1994). 